\title{
Die Republik der Gelehrten 2.0
}

Der öffentliche Diskurs und der Austausch unter Spezialistinnen und Spezialisten verlagern sich immer mehr in den virtuellen Raum. Das sogenannte Web 2.0 bietet der Disziplin der Internationalen Beziehungen (IB) neue Möglichkeiten der internen und externen Kommunikation und verändert den Wissenschaftsbetrieb. Die Kommunikation ist schnell, dezentral und richtet sich an eine zuvor schwer erreichbare Öffentlichkeit. Dies birgt Risiken und Chancen. Statt diese neuen Möglichkeiten zu nutzen und das Profil des Fachs in der öffentlichen Wahrnehmung und der Politik zu schärfen, verliert man mit Blick auf die Risiken die Chancen aus den Augen. Die Disziplin riskiert dabei, den Graben zu den englischsprachigen IB zu vergrößern und an internationaler Relevanz zu verlieren. Die Neuauflage der Republik der Gelehrten könnte so ohne die deutschsprachige Welt stattfinden.

\section{Die Republik der Gelehrten $2.0^{1}$}

Ein interdisziplinäres Team aus den Computer- und Geschichtswissenschaften entwickelte 2009 an der Universität Stanford eine Visualisierung des intensiven Briefverkehrs zwischen Gelehrten der Aufklärung (Chang et al. 2009). Dieser Briefwechsel erstreckte sich über ganz Europa, bis nach Indien und über den Atlantik nach Nordamerika. Er ist eine Facette eines Netzwerkes, das sich über Briefe hinaus auch in Salons, Kaffeehäusern, Freundschaften und Vorträgen in direkten Kontakten manifestierte. Nutzt man das vom Stanford Team geschaffene interaktive Programm, entsteht ein eindrückliches Bild einer vernetzten intellektuellen Gemeinschaft, die nationale Grenzen hinter sich ließ und durch einen regen Austausch von Ideen sich im 18. Jahrhundert einen eigenen geistigen Raum schaffte. Wir kennen dieses Netzwerk heute unter der Bezeichnung der Republik der Gelehrten. Die philosophes (wie diese Denker und Denkerinnen damals bezeichnet wurden), haben auf diesem Weg Wissenschaft, Politik und Geschichte nachhaltig bis in die heutige Zeit beeinflusst. Eine Visualisierung dieses Netzes hat eine faszinierende Ähnlichkeit mit Darstellungen von Internet-Traffic und internationaler digitale Vernetzung (Harrison 2007). Dies kommt nicht von ungefähr.

Das Internet verspricht uns eine neue Republik der Gelehrten, vernetzt jenseits der kühnsten Phantasien der Bürgerinnen und Bürger der alten Republik der Gelehrten. Die Werkzeuge des sogenannten Web 2.0 ersetzen die bisherige Funktionsweise des akademischen Austausches zwar nicht, bieten jedoch unzählige neue

1 Ich möchte Mathis Lohaus und den anonymen Gutachterinnen/Gutachtern für die nützlichen und konstruktiven Kommentare danken. Fehler und Auslassungen sind meine eigenen. 
Möglichkeiten. Diese Republik der Gelehrten 2.0 eröffnet die Aussicht auf eine engere, aktuellere und weitläufigere Vernetzung, die man sich bisher kaum vorstellen konnte. Eines dieser Instrumente des Webs 2.0 sind sogenannte Weblogs oder kurz Blogs. Ich wurde gebeten, über die Rolle von Blogs im wissenschaftlichen Diskurs in den Internationalen Beziehungen zu reflektieren. Ich werde betrachten, inwieweit Blogs sich für das Fach der Internationalen Beziehungen als Werkzeug eignen, wo deren Potential liegt und ob sie entsprechend genutzt werden. Ich postuliere drei Thesen zu Blogs und unserer Disziplin in diesem Beitrag: Erstens, Blogs werden in Zukunft eine wichtige Rolle im wissenschaftlichen Diskurs spielen, egal ob man das für wünschenswert hält oder nicht. Darum müssen wir uns damit befassen. Zweitens, die Disziplin der Internationalen Beziehungen ist besser geeignet als viele andere sozialwissenschaftlichen Fächer, um von Blogs zu profitieren. Drittens und vielleicht etwas provokant, die deutschsprachige IB-Welt ist gerade dabei, diese Chance zu verschlafen und sich so ins Abseits zu manövrieren.

In den letzten Jahren hat sich eine lebendige akademische Blogszene im Netz entwickelt. Inzwischen werden diverse Wissenschaftsblogportale durch größere Verlage betrieben und haben einen festen Platz in deren Onlineangebot. Trotz dieser Etablierung fällt auf, dass Wissenschaftsblogs mehrheitlich naturwissenschaftlich sind, und dass Blogs im englischsprachigen Raum (vor allem den USA) viel mehr zum akademischen Alltag gehören. Blogs werden dort als Medium anscheinend ernster genommen, manchmal sogar als eine vorgelagerte Phase des peer review-Prozesses.

Es geht in diesem Beitrag nicht darum, in naiver Technikverblendung in den Chor jener einzustimmen, die im Web 2.0 nur eine verklärte Zukunft sehen können. Blogs im Wissenschaftsbetrieb bringen auch Nachteile mit sich und das stelle ich nicht in Abrede. Es geht viel mehr darum, den potentiellen Nutzen dagegen abzuwägen. Im Moment werden Blogs im deutschsprachigen Raum in unserer Disziplin im besten Fall nicht beachtet, im schlimmsten Fall als etwas Negatives, die wissenschaftliche Arbeit Behinderndes, gesehen. Statt Forschende zu motivieren, die Möglichkeiten des Webs 2.0, die bei vielen privat zum Alltag gehören, beruflich zu nutzen, werden Anreize geschaffen, diese Kommunikations- und Arbeitsinstrumente in der Arbeit zu meiden. Dies schränkt nicht nur ein, wie geforscht wird, sondern ist längerfristig schädlich für die Disziplin der Internationalen Beziehungen. Um unnötige Abwehrreflexe hinter uns zu lassen und die neuen Möglichkeiten für das Fach zu nutzen, muss eine Diskussion geführt und die Skepsis überwunden werden. Ich hoffe, dieser Artikel kann einen kleinen Beitrag dazu leisten.

Ich werde in diesem Artikel versuchen, das Phänomen der Blogosphäre als virtuelles Kaffeehaus der neuen Republik der Gelehrten auszuleuchten, um herauszufinden, ob für die Internationalen Beziehungen dort ein Tisch frei ist und wo dieser steht. Der Beitrag ist hierfür in drei Teile gegliedert: In einem ersten Teil werde ich für die Lesenden, die mit dem Medium Blog nicht vertraut sind, eine kleine tour d'horizon zu Wissenschaftsblogging im Allgemeinen und den Sozialwissenschaften im Speziellen anbieten. Im darauf folgenden Teil werde ich mich dann spezifisch mit der Disziplin der Internationalen Beziehungen und Blogs beschäftigen. Der 
dritte Teil fokussiert auf die Konsequenzen der aktuellen Nutzung von Blogs in unserem Fach.

\section{Blogs und Wissenschaft}

Vermutlich sind nicht alle Leserinnen und Leser vertraut mit dem Medium Blog. Zumindest wenn meine These, dass sich unsere Disziplin bisher nicht ausreichend mit diesen auseinandersetzt, korrekt ist, dann sollte ich diesen ersten Teil mit einer kleinen Einführung beginnen. Jene, die mit Blogs vertraut sind, können die nächsten zwei Abschnitte problemlos überspringen. Die Wikipedia (und wann wäre es je angebrachter, diese als Quelle zu benutzen) liefert folgend Etymologie und Definition:

»Das Blog (auch: der Blog) oder auch Weblog, engl. Wortkreuzung aus engl. World Wide Web und Log für Logbuch, ist ein auf einer Website geführtes und damit - meist öffentlich - einsehbares Tagebuch oder Journal, in dem mindestens eine Person, der WebLogger, kurz Blogger genannt, Aufzeichnungen führt, Sachverhalte protokolliert oder Gedanken niederschreibt.« (Wikipedia Deutschland 2014)

Es handelt sich also um eine meist öffentlich zugängliche Internetseite, auf der mit Datum versehene Einträge erstellt werden. Diese Einträge, die von ein paar Zeilen bis zu Magazin-Artikellänge reichen können, bieten die Möglichkeit, via Hyperlinks auf andere Texte im Netz zu verweisen. Die meisten Plattformen bieten die Möglichkeit, Einträge direkt zu kommentieren. Diese Funktion kann nach Belieben deaktiviert oder eingeschränkt werden (z.B. mit einer Registrierungspflicht).

Hier bietet sich auch schon eine erste Gelegenheit, mit einem Vorurteil gegenüber Blogs aufzuräumen. Die Tagebuchelemente verleiten viele dazu, Blogs als persönliche Schreibübungen im Netz zu betrachten, irgendwo oszillierend zwischen einem Narzissmus-Projekt und einer Form von Exhibitionismus. Diesen Typus gibt es zwar durchaus in den Weiten des Internets (und es gibt auch eine Tagebuchform des Wissenschaftsbloggings, dazu aber später mehr), dieser Beitrag bezieht sich jedoch primär auf die Welt der Wissenschafts- und Politikblogs, die in der Regel mehr sind als eine persönliche digitale Nabelschau einer Bloggerin oder eines Bloggers.

Eine Stärke des Mediums ist seine Vielseitigkeit. Um zu verstehen, wie Blogs im akademischen Bereich genutzt werden können oder wo ihre Nachteile liegen, muss zuerst geklärt werden, auf welche Arten sie für die wissenschaftliche Arbeit einsetzbar sind. Dieser Nutzen beschränkt sich nicht auf die Internationalen Beziehungen oder die Sozialwissenschaften. Das Medium ist für die akademische Arbeit an und für sich vielversprechend. Auf den speziellen Nutzen für die Disziplin der Internationalen Beziehungen werde ich später nochmals zurückkommen. Es gibt vier Einsatzmöglichkeiten von Blogs, die ich hier diskutieren möchte: als didaktisches Hilfsmittel in der Lehre, für den disziplininternen Austausch (der Kern der neuen Republik der Gelehrten), für Kommunikation nach Außen mit einer breiteren Öffentlichkeit und last but not least, um Expertise und Fachwissen in den aktuellen 
politischen Diskurs einzubringen. Ich werde kurz auf jede dieser Nutzungen und die damit verbundenen Vor- und Nachteilen eingehen.

Ich beginne mit dem Einsatz von Blogs in der Lehre. Die Nutzung von Blogs im Unterricht ist für diesen Beitrag weniger von Bedeutung, sollte aber trotzdem vollständigkeitshalber erwähnt werden (vgl. hierzu auch den Beitrag von Quaintance im selben Forum). Das Blog als didaktisches Werkzeug wurde schon viel diskutiert und der potentielle Nutzen in der Lehre von kompetenterer Seite besprochen (vgl. hierzu auch den Beitrag von Quaintance im selben Forum; vgl. außerdem Hidgon/ Topaz 2009; Churchill 2009; Brownstein/Klein 2006; Krause 2005; Ferdig/Trammell 2004). Blogs können als Diskussionsplattform, Kooperationsübung, klasseninternes peer review, als Schreibübung oder Publikationsplattform genutzt werden. Man kann so den Studierenden gleichzeitig Methodisches, Inhalte und eine Vertrautheit mit dem Medium vermitteln. Natürlich ist es mit dem bloßen Einsatz von Blogs im Unterricht nicht getan. Wie andere Hilfsmittel auch, muss der Einsatz zielgerichtet sein und es müssen die richtigen Rahmenbedingungen bestehen. Zudem stellen Blogs nur ein Element des Web 2.0 dar und es gibt viele andere Instrumente, die nicht vernachlässigt werden sollten (z.B. Twitter, RSS, Online-Publikationen etc.).

Die zweite nützliche Funktion von Blogs für die Wissenschaft liegt in den Möglichkeiten zum disziplinären und auch interdisziplinären Austausch. Es handelt sich um den Kern der Republik der Gelehrten 2.0. Ich möchte diesbezüglich vor allem drei Aspekte hervorheben: Blogs können erstens eine Form eines informellen peer review sein. Sie können diesem Prozess vor- oder nachgelagert sein. Dank einfachem Einstieg und Nutzung können Blogs zweitens eine Plattform für den regelmäBigen Austausch von Spezialistinnen und Spezialisten bieten, aber auch disziplinäre Schranken überwinden helfen. Drittens können Blogs sich als nützlich erweisen, um den impact der eigenen Forschung zu erhöhen und das eigene Forschungsprofil zu schärfen. Diese drei Aspekte des Austausches sollen hier kurz zur Sprache kommen.

Beginnen wir mit Blogs als Erweiterung des peer review-Prozesses. Viele Wissenschaftsbloggerinnen und -blogger verwenden Blogs als digitale Notizblöcke. Da diese Notizen in den öffentlichen Raum »gehängt« werden, ermöglichen sie, ähnlich dem Vorstellen einer ersten Fassung eines Projektes auf einer Konferenz, Ideen zu präsentieren und noch Unausgegorenes zur Diskussion zu stellen. Rückmeldungen können so viel schneller und unmittelbarer kommuniziert werden als mit den herkömmlichen Methoden. Ein Fachpublikum kann so auch forschungsrelevante Tagesaktualität diskutieren. Blogs können nicht nur ergänzend, sondern auch als Korrektive zum herkömmlichen peer review-Prozess dienen. Dazu ein Beispiel aus den Naturwissenschaften: Als 2010 das renommierte Journal Science einen Aufsatz veröffentlichte, in dem NASA-Forscherinnen und -Forscher behaupteten, auf Arsen basierende Lebensformen gefunden zu haben (Wolfe-Simone et al. 2010), wurde diese Entdeckung sofort in diversen wissenschaftlichen Blogs und auf dem Kurznachrichtendienst Twitter scharf kritisiert. Diverse Medien griffen diese Kritik auf (Shema et al. 2012) und es bestehen heute tatsächlich große Zweifel an der Aussa- 
gekraft der Resultate. Die Kritik hatte die regulären Medien erreicht, bevor Science überhaupt technische Kommentare zum Artikel, einer davon von einem Blogger, hochladen konnte (Shema et al. 2012).

Blogs können zweitens die Kommunikation erleichtern, indem sie dem direkten Austausch unter Expertinnen und Experten dienen. Diskussionen, die sonst nur auf internationalen Forschungskonferenzen stattfinden, sind plötzlich 365 Tage im Jahr möglich. Zusätzlich findet eine Demokratisierung statt, da wegen der möglichen Anonymität der Inhalt der Kommentare mehr ins Zentrum rückt. Ein gutes Beispiel hierfür liefern Charlie Carpenter und Daniel Drezner in ihrem 2010 erschienenen Aufsatz zum Web 2.0 und den Internationalen Beziehungen: Ein IB-Gemeinschaftsblog zögerte demnach zuerst, eine für gut befundene Webseite permanent im Blog zu verlinken, da sich herausstellte, dass es sich bei der Verfasserin um eine Studierende handelte. Trotz Erfahrungsmangel wurde das Blog schließlich aufgenommen, weil »das Material für sich selbst sprechen soll« (Carpenter/Drezner 2010: 267). Spezialisierte Blogs können auch helfen, die so oft herbeigewünschte, aber weniger oft gelebte Interdisziplinarität zu realisieren und andere fachliche Perspektiven einzubringen. Gerade bei Blogportalen, die naturwissenschaftliche und sozialwissenschaftliche Blogs vereinen, ist das sehr interessant. ${ }^{2}$

Drittens kann im Rahmen von wissenschaftsinterner Kommunikation ein wissenschaftliches Blog eine unverzichtbare virtuelle Visitenkarte von Forschenden sein. Die London School of Economics hat etwa ein Blog online, das sich vor allem der Frage widmet, wie die Werkzeuge des Web 2.0 dafür genutzt werden können, die eigene sozialwissenschaftliche Forschung besser zu bewerben (LSE Impact Blog). Dazu hat das LSE Impact Blog ein Online-Handbuch verfasst mit Tipps, wie die Sichtbarkeit von Publikationen erhöht und die Qualität unter anderem durch das Schreiben eines Blogs und Präsenz in anderen sozialen Medien verbessert werden kann (LSE Impact Blog 2011). Ein regelmäßig aktualisiertes Blog kann bei der Positionierung eigener Forschung helfen. Natürlich muss die Qualität stimmen, denn auch Negatives ist in der Vitrine des Internets für fast immer ausgestellt und auffindbar. Durch Bloggen kann auch die Platzierung der eigenen Forschung in den von Suchmaschinen gelieferten Resultaten verbessert werden. In Kombinationen mit anderen Instrumenten, wie dem Kurznachrichtendienst Twitter, kann man so einfach und effektiv ein interessiertes, spezialisiertes Publikum auf Publikationen aufmerksam machen.

Natürlich ersetzen Blogs die herkömmlichen Mittel der Interaktion nicht. Es gibt nach wie vor eine wichtige Rolle für den klassischen pee review-Prozess. Weder das persönliche Element einer wissenschaftlichen Konferenz, noch eines Autorinnen/Autoren-Workshops kann damit ersetzt werden. Es schafft auch nicht die Notwendigkeit von herkömmlichen Publikationen ab. Es ist eine nützliche Ergänzung zur traditionellen Forschungsarbeit, die bestimmte Pfeiler des Wissenschaftsbetriebs, wie die soeben genannten, bereichert.

2 Im deutschsprachigen Raum geschieht das beispielsweise auf scilogs.de und scienceblogs.de. 
Es gibt eine dritte wichtige Funktion von Wissenschaftsblogs, die hier diskutiert werden muss: Die Kommunikation nach außen. Das Verlassen des Elfenbeinturms fällt nicht allen Forschenden leicht. Zwischen Publikationsdruck, administrativen Pflichten und Lehre wird der Wissenschaftskommunikation oft eine niedrige Priorität im Pflichtenheft beigemessen. Dies ist in zweierlei Hinsicht bedauernswert. Einerseits existiert durchaus ein breites Interesse, sich mit der Welt der Wissenschaft auseinanderzusetzen. Dies zeigen nicht zuletzt die Erfolge von diversen wissenschaftlichen Blogportalen in verschiedenen Sprachen und Ländern. Andererseits ist es ein wichtiges und notwendiges Element der Öffentlichkeitsarbeit, das langfristig ein zentrales Interesse der Wissenschaft sein sollte.

Zweifelsohne existiert eine Nachfrage nach Nachrichten aus der Wissenschaft. Es existieren mehrere Zeitschriften, die diese Nische bedienen (zugegebenermaßen mehr in den Natur- als in den Sozialwissenschaften). Die meisten nicht auf wissenschaftliche Berichterstattung spezialisierte Medien haben einen Wissenschaftsteil, in dem oft aufgrund knapper Ressourcen wöchentlich dieselbe Handvoll Pressemeldungen, die überall zu finden sind, verarbeitet werden. Blogs sind da ein zusätzliches Angebot und ein Fenster zu spezialisierten Nischen. Nischen, die vielleicht ansonsten gar keine Öffentlichkeit erreichen würden. Es gibt mehrere (anekdotische) Hinweise, dass dank einer Veröffentlichung in Blogs, auch im deutschsprachigen Raum Forschung von regulären Medien erst aufgegriffen wurde.

Aber Bloggen für die Wissenschaftskommunikation ist mehr als eine reine Gratis-Dienstleistung. Es handelt sich gerade für die Sozialwissenschaften um eine Notwendigkeit. Da viel Forschung an Universitäten von öffentlichen Geldern finanziert wird, stellen sich einige auf den Standpunkt, dass ein Vermitteln von Forschungsresultaten eigentlich eine Pflicht für Forschende darstellt. Obwohl diskutiert werden kann, ob tatsächlich eine Bringschuld besteht, ist es auf jeden Fall schwer, in Abrede zu stellen, dass solche Öffentlichkeitsarbeit im Interesse der Forschungsgemeinschaft ist. Es geht auch um Public Relations und Lobbying für die Disziplin. Der Vorstoß im US-Kongress des Senators Tom Coburn vom März 2013 kann als Warnung vor den möglichen Konsequenzen einer vernachlässigten Öffentlichkeitsarbeit dienen. Der vom US-Senat akzeptierte Vorschlag verunmöglichte der National Science Foundation nahezu, Projekte aus den Politikwissenschaften zu unterstützen. Der Vorstoß betraf ausschließlich politikwissenschaftliche Eingaben (mit der Ausnahme solcher, die für die nationale Sicherheit von Bedeutung sind). Kurzzeitig wurde die staatliche Finanzierung von Projekten in der Disziplin über die National Science Foundation wesentlich erschwert (Basken 2013). Die Regelung wurde glücklicherweise in der Zwischenzeit wieder zurückgezogen. Nicht zuletzt Blogs können einen wichtigen Beitrag zu einer besseren Öffentlichkeitsarbeit leisten und die Public Relations für unsere Disziplin nachhaltig stärken.

Blogs sind gut geeignet für diese Art von Kommunikation. Weil viele Blogs auch immer wieder Platz für etwas persönlichere Notizen bieten, sind sie unmittelbarer als die reguläre Wissenschaftsberichterstattung und können so der Wissenschaft ein Gesicht geben. Es gibt auch Blogs, die zurück zur ursprünglichen Tagebuchform gefunden haben und im Stile eines Laborjournals den Forschungsalltag einem brei- 
teren Publikum vermitteln. Beispiele dafür sind unter anderem auf der französischen Plattform hypotheses.org zu finden, wo sich zum Beispiel die archäologischen Ausgrabungsjournale großer Beliebtheit bei den Lesenden erfreuen (Hypotheses 2014).

Zum Schluss noch die vierte, für die Internationalen Beziehungen vielleicht interessanteste Funktion von Blogs: Sie sind eine Möglichkeit, sich in den politischen Diskurs einzumischen. In Zeiten, wo in Nachrichtensendungen und Talkshows echten und vermeintlichen Expertinnen respektive Experten viel Raum gegeben wird, scheint eine Einmischung mit Fachwissen zentral und das Beisteuern von Evidenz notwendig. Man könnte argumentieren, es ist ein wichtiger Beitrag zu einer funktionierenden Demokratie. In den USA haben Blogs, wie zum Beispiel jener des Wirtschaftsnobelpreisträgers Paul Krugman, einen festen Platz in der Meinungsbildung und dem politischen Diskurs. (The Conscience of a Liberal 2014) Solche public intellectuals (oder vielleicht sollte man sagen philosophes) sollen auch aus unserem Fachbereich kommen. Außenpolitik nimmt im Newszyklus eine zentrale Rolle ein und ist dank Phänomenen wie Globalisierung oder supranationalen Institutionen wie jene der Europäischen Union immer mehr auch Innenpolitik. Hier sollte sich die Disziplin einbringen und auch so die Relevanz unserer Forschung belegen. IB-Blogs können einen Beitrag leisten, die Qualität der politischen Deliberation aufzuwerten und hoffentlich auf diese Art zu besseren Entscheidungen verhelfen.

Diese vier Funktionen wissenschaftlicher Blogs (Lehre, interne Kommunikation, externe Kommunikation, Politikdiskurs) bieten sich den Naturwissenschaften genau so wie den Sozialwissenschaften an. Inwiefern diese Möglichkeiten genutzt und wie effektiv sie in der Disziplin der Internationalen Beziehungen eingesetzt werden, soll Thema des nächsten Kapitels sein.

\section{Die IB-Blogosphäre}

Das Potential und die Möglichkeiten des Wissenschaftsbloggings sind also vielfältig. Nun stellt sich die Frage nach der effektiven Nutzung von Blogs in den Internationalen Beziehungen. Dazu werde ich hier einen Einblick in die einschlägige Blogosphäre zu geben versuchen. Ich beginne mit einem Überblick anhand von Beispielen (ohne Anspruch auf Vollständigkeit). Dieser wird sich nicht nur auf deutschsprachige Blogs beschränken, aus gutem Grund, wie wir sehen werden. Daraus ergeben sich Fragen nach den Gründen für die unterschiedliche Nutzung, aber auch nach dem Einfluss von Blogs in der Disziplin. Es ist weder das Ziel noch praktisch machbar, im Rahmen dieses Forumsbeitrages eine detaillierte empirische Studie zu Blogs zu liefern. Es existieren leider auch nur wenige Untersuchungen dazu und für die Internationale Beziehungen gibt es solche meines Wissens nicht.

Wenn wir zuerst einen Blick auf sozialwissenschaftliche Blogs ganz allgemein im Netz werfen, stellt man fest, dass die Wissenschaftsblogosphäre naturwissenschaftlich dominiert ist. Shema et al. (2012) bestätigen dies in ihrer kleinen Studie 
zur Webplattform researchblogging.org. Es handelt sich um einen Aggregator für Beiträge in Blogs über wissenschaftliche Fachartikel. Man kann sich als Blogbetreiberin respektive -betreiber dort anmelden. Blogeinträge zu Aufsätzen in wissenschaftlichen Zeitschriften, die den peer review-Prozess durchlaufen haben, werden dann auf dem Portal automatisch verlinkt. Die Seite ist somit gleichzeitig Informationsknotenpunkt für Wissenschaftsblogging und eine Art label für Beiträge über Forschung, die einen gewissen Qualitätsstandard erfüllen. Shema et al. fanden, dass nur 5 Prozent der dort registrierten Wissenschaftsblogs sich in den Sozial- und Geisteswissenschaften einordnen, während 39 Prozent naturwissenschaftliche Blogs waren. An zweiter Stelle finden wir die Kategorie Psychologie, Psychiatrie, Neurowissenschaften und Verhaltensforschung. In diesen Bereichen existiert auch eine Überlappung mit den Sozialwissenschaften.

Sucht man nach sozialwissenschaftlichen Beiträgen von Blogs auf Deutsch in researchblogging.org ist das Bild noch mehr von der Abwesenheit solcher geprägt. Es gibt kaum Beiträge in dieser Kategorie und die große Mehrheit stammt von Naturwissenschafts-Blogs, die ein Schnittstellenthema behandelt haben. Nun gewährt dies nur einen sehr beschränkten und vielleicht nicht sehr repräsentativen Einblick in die deutschsprachige, sozialwissenschaftliche Blogosphäre. Wie schon erwähnt, war das Portal von Anfang an eher naturwissenschaftlich dominiert. Es wird zudem auch bei Naturwissenschaftlerinnen und -wissenschaftlern nur von einer Untergruppe genutzt.

Es gibt sie natürlich schon, die sozialwissenschaftlichen Blogs in den deutschsprachigen Ecken des Internets. Es sind nicht Massen, aber man findet doch z.B. einige Soziologie-Blogs auf Deutsch. Die nicht mehr erreichbare Webseite Wissenschafts-Café, übrigens von einem Soziologen betrieben, listet unter der Kategorie Sozialwissenschaften rund 63 deutschsprachige Blogs auf (einige davon wurden länger nicht mehr aktualisiert). Es gilt dabei ebenfalls zu beachten, dass bei dieser Zählung Blogportale nur einmal gelistet und somit als nur ein Blog gezählt werden. Die größten deutschsprachigen Wissenschaftsblogportale scilogs.de und scienceblogs.de beherbergen etwa 50 respektive 30 aktive Blogs. ${ }^{3}$ Dazu gehören trotz der naturwissenschaftlichen Ausrichtung auch einige bloggende Sozial- und Geisteswissenschaftlerinnen und -wissenschaftler. Es fällt dabei auf, dass das Spektrum von nicht-naturwissenschaftlichen Blogs breit gefächert ist, von Theologie bis zur Psychologie oder Geographie und dass Blogs klassischer sozialwissenschaftlicher Ausrichtung an einer Hand abgezählt werden können. Das offene Blogportal hypotheses.org listet 53 deutschsprachige Blogs. Es gibt zudem auch ein paar wenige institutionelle Blogs von Universitäten oder anderen akademischen Institutionen (z.B. führt die Deutsche Gesellschaft für Soziologie ein Gemeinschaftsblog), diese sind aber relativ selten, und viele davon dienen eher als internes Anschlagsbrett und nicht als Kommunikationsangebot an eine breitere Öffentlichkeit.

3 Scilogs.de (Scilogs 2014) ist eine Plattform, die von der populärwissenschaftlichen Zeitschrift Spektrum der Wissenschaft betrieben wird, scienceblogs.de (Scienceblogs 2014) gehörte bis vor kurzem dem US-Verlag National Geographic und wurde nun an die Konradin Mediengruppe, die u.a. Bild der Wissenschaft herausgibt, verkauft. 
Wie sieht die Blogosphäre in den Internationalen Beziehungen aus? Eine einfache Suchanfrage bei Google auf Deutsch zu Internationale Beziehungen und Blog fördert nicht sehr viel zu Tage. Institutionelle Blogs finden sich auf der ersten Seite gar keine. Das erste solche Blog findet sich mit einer Sammlung an Stellenangeboten - sicherheitspolitik-blog.de (Sicherheitsbpolitik Blog 2014) - und taucht erst auf der zweiten Resultatseite auf. Es finden sich vielleicht drei Blogs geschrieben von Personen mit einem IB-Hintergrund, alle scheinen individuelle Initiativen zu sein. Die Tatsache, dass viele Hits auf Seiten zur studentischen Mobilität verweisen, ist wohl auch ein Indikator dafür, dass es für die Disziplin der Internationalen Beziehungen zumindest in Bezug auf Suchmaschinenoptimierung noch viel unerledigte Arbeit gibt.

Nun gibt es durchaus eine Präsenz von Blogs zu internationalen Beziehungen aus dem deutschsprachigen Raum. Diese scheinen aber nur eine kleine Nische zu besetzen und sind rar. Es gibt relativ neue und wenig etablierte studentische Projekte mit dem Bretterblog (2014) oder dem IR-Blog (2014), wobei letzterer bisher ausschließlich auf Englisch verfasst wird. Seit rund sieben Jahren gibt es zudem das vom Autor dieses Beitrages geführte zoon politikon - ein IB-Blog auf dem Portal der scienceblogs.de (Zoon politikon 2014). Dieses ist aber in ein disziplinfremdes Portal eingebunden und behandelt deshalb des Öfteren andere Themen als die Internationalen Beziehungen. Den Rückgriff auf die englische Sprache macht auch das ausschließlich auf Englisch geführte ISN-Blog der Eidgenössischen Technischen Hochschule Zürich (International Relations and Security Network 2014). Professorinnen oder Professoren, die bloggen, findet man nahezu nicht im deutschsprachigen Raum. Die einzigen mir bekannten Ausnahmen sind vereinzelte Beiträge auf dem Sicherheitspolitik-Blog, welches gemäß Impressum »zur Professur für Internationale Organisationen « (Sicherheitspolitik-Blog 2014) an der Universität Frankfurt gehört. ${ }^{4}$ Es existieren ein paar wenige Ausnahmen in den benachbarten Disziplinen der Politischen Wissenschaften. ${ }^{5}$ Auch gibt es Initiativen wie das bloggingportal.eu, welches aber eher politische Kommentare ohne bedeutenden akademischen Anspruch bietet. Dieser Überblick hat natürlich keinen Anspruch auf Vollständigkeit. Die Tatsache, dass es schwierig ist, IB-Blogs auf Deutsch zu finden, heißt natürlich nicht, dass sie nicht existieren. Ein Blick auf die blogrolls ${ }^{6}$ der deutschsprachigen IB-Blogs untermauert den ersten Eindruck jedoch. Im großen Ganzen kann man feststellen, dass dem Bloggen in der Disziplin kein großer Stellenwert beigemessen wird. Ist die Republik der Gelehrten 2.0 also nur eine technikverliebte Idee

4 Der Inhaber des Lehrstuhls Professor Christopher Daase schreibt auf dem Gemeinschaftsblog regelmäßig Beiträge.

5 Zum Beispiel der Soziologe Günther Voß, der zum SozBlog (SozBlog 2014) beiträgt, das PoliSciBlog der Universität Zürich (PoliSciZürich 2014) wo regelmäßig Professorinnen und Professoren bloggen oder der Schweizer Politologe Claude Longchamps (Zoon politicon 2014).

6 Listen von Links zu anderen Blogs die typischerweise auf Blogs geführt werden. Es sind eine Art Leseempfehlungen und ein gute Methode neue (vor allem spezialisierte) Blogs zu entdecken. 
ohne echte Chance auf Realisierung in den Internationalen Beziehungen? Nicht ganz.

Wenden wir uns also der englischsprachigen Blogging-Szene in der Disziplin zu. Zuerst gibt es natürlich die schon erwähnten Blogs im deutschsprachigen Raum, die in Englisch verfasst werden. Richtet man jedoch seinen Blick über den Atlantik, zeigt sich ein anderes Bild von Präsenz und Vernetzung. Der Vergleich ist natürlich teilweise unfair. Englisch ist die Lingua franca der Disziplin und will man durch Bloggen das Profil der Institution oder das eigene Forschungsprofil erhöhen, dann drängt es sich auf, auf Englisch zu bloggen. Ganz abgesehen davon, dass Zielpublikum und Vernetzungspotential viel größer sind, weil das Fach der Internationalen Beziehungen im englischsprachigen Raum über eine größere Tradition verfügt.

Die beste Sichtbarkeit haben wohl Gemeinschaftsblogs und Blogs auf größeren Portalen. Das vermutlich meistgelesenste wissenschaftliche Blog der Internationalen Beziehungen ist Duck of Minerva (Duck of Minerva 2014), ${ }^{7}$ wo man Diskussionen zur aktuellen Forschung findet, aber auch oft theoretische Fragen aufgeworfen werden, Debatten zum Stand der Disziplin geführt oder praktische Tipps für Konferenzteilnahmen oder dem Verfassen von akademischen Lebensläufen gegeben werden. Auf der anderen Seite finden wir Zeitschriften wie Foreign Policy, die als Blogportale fungieren und Persönlichkeiten aus der Disziplin angeworben haben. So bloggen auf foreignpolicy.com unter anderem die beiden Professoren Stephen Walt und Daniel Drezner (Drezner Foreign Policy 2014; Walt Foreign Policy 2014). Ebenfalls eine große Reichweite dürfte das Monkey-Cage-Blog haben, welches oft Beiträge aus den Internationalen Beziehungen veröffentlicht und inzwischen bei der Washington Post zu Hause ist (Monkey Cage 2014). Dies sind nur drei Beispiele von sehr sichtbaren IB-Blogs auf Englisch. Es gibt unzählige mehr: Blogs von universitären Einreichtungen, Individualblogs, thematische Blogs zu spezifischen Weltregionen oder Subthemen wie Internationale Politische Ökonomie, Abrüstung und viele mehr. Alle diese hier aufzulisten, würde den Rahmen dieses Beitrages sprengen, und jede Auswahl wäre zumindest partiell willkürlich.

Drezner ist wie schon erwähnt auch Co-Autor von Carpenter in der ebenfalls schon erwähnten Publikation im International Studies Quarterly (ISQ), die sich mit dem Nutzen des Webs 2.0 für die Disziplin auseinandersetzt. Carpenter und Drezner stellen für die viel weiter entwickelte englischsprachige IB-Blogosphäre fest, dass auch dort die notwendige Diskussion über Blogs nicht oder kaum stattfindet: »To date, there has been a decided lack of scholarly discussion about the ways in which Web 2.0 affects the discipline, pedagogy, or subject matter of IR « (Carpenter/Drezner 2010: 256). Diese Diskussion findet leider auch auf Deutsch nicht statt. Im Gegensatz zum englischen Sprachraum scheint hier aber auch kaum jemand daran Interesse zu haben. Dies könnte längerfristig zu einem großen Problem der Internationalen Beziehungen in Deutschland werden. Selbst in den USA, wo es eine

7 Genaue Zahlen zu Leserinnen und Leser sind schwer zu bekommen, gerade auch für kommerzielle Portale wie zum Beispiel Foreign Policy (Foreign Policy Magazine 2014). Diese werden meist als Geschäftsgeheimnis behandelt. 
viel lebendigere IB-Blogging-Szene gibt, macht sich nach wie vor Widerstand bemerkbar. So gab es vor kurzem ein Blog-Verbot für Redakteurinnen und Redakteure der hauseigenen Publikationen auf der Traktandenliste des Governing Council der International Studies Association (ISA). Der eher unausgegorene Vorschlag wurde nach einem Proteststurm in der IB-Blogosphäre - ein Proteststurm, der auch traditionelle Medien in Übersee wie den britischen Guardian erreichte (Greenslade 2014) - vorläufig auf Eis gelegt, um »mehr Rückmeldungen zu sammeln« (Straumsheim 2014).

\section{Verpasste Chancen?}

Im ersten Teil habe ich allgemein über den potentiellen Nutzen von Wissenschaftsblogging geschrieben, um danach in einem zweiten Teil einen Einblick zu existierenden Blogs in den Internationalen Beziehungen zu geben. Dabei zeichnet sich ein Rückstand der deutschsprachigen IB-Blogosphäre ab. Die Frage stellt sich natürlich, inwiefern dies ein Problem ist. Welchen spezifischen Nutzen bringt Bloggen den IB? Die Beantwortung dieser Fragen hilft bei der Evaluation, was die konstatierte Blog-Aversion im deutschsprachigen Raum in unserer Disziplin für Konsequenzen haben könnte. Dazu möchte ich die vier Kategorien wieder aufnehmen, die ich zu Anfang als die vier Vorteile von Wissenschaftsblogging aufgelistet habe und sie mit Blick auf die Internationalen Beziehungen diskutieren. Zur Erinnerung, die vier Elemente waren: Lehre, akademischer Austausch, Kommunikation nach außen und der politische Diskurs.

Beginnen wir mit der Nutzung von Blogs in der Lehre. Es ist anzunehmen, dass Blogs in der Disziplin der Internationalen Beziehungen in dieser Art kaum genutzt werden. Es wäre erstaunlich, wenn beim mangelnden Enthusiasmus, der für dieses Medium im deutschsprachigen Raum besteht, es trotzdem systematisch in der Lehre eingesetzt würde. Dies scheint im Moment zumindest nur bedingt problematisch. Man verzichtet auf eine Option unter vielen, den Stoff zu vermitteln. Es handelt sich um ein zusätzliches, aber nicht unbedingt notwendiges Instrument. Es ist auch schwer zu sagen, wie oft Blogs in der Lehre im englischsprachigen Raum genutzt werden. Sie tauchen selten auf Syllabi auf, und wenn sie es tun, dann meistens im Unterricht von Forschenden, die selber mehr oder weniger prominent bloggen. Die Kluft scheint hier nicht allzu groß zu sein.

Problematisch ist jedoch, dass eine Generation von Studentinnen und Studentinnen das Potential ebenfalls nicht entdecken kann und so der Eindruck implizit vermittelt wird, dass Blogs nicht akademisch und nicht seriös genug für die Forschung sind. Die Berührungsängste mit Blogs werden so in der Disziplin perpetuiert. Selbst dem Bloggen gegenüber skeptisch eingestellte Leserinnen und Leser können sicherlich nachvollziehen, dass damit ein unter Umständen nützliches und vielseitiges didaktisches Werkzeug von vorneweg ausgeschlossen wird.

Die Probleme sind noch pointierter, wenn es um den internen Austausch geht. Viele der von mir beschriebenen potentiellen Vorteile des Bloggens sind Blogs von 
Individuen: Es geht um die Aufmerksamkeit für die eigene Forschung, Kommentare und Anregungen von peers, Anknüpfungspunkte für Kontakte, Namenserkennung, etc. Gerade für Nachwuchsforscherinnen und -forscher sind dies wichtige Aspekte für ihre Karriere. Blogs scheinen in verschiedenen Disziplinen zunehmend zur Grundausbildung in der englischsprachigen akademischen Welt zu gehören. Wenn dieses Wissen und diese Fähigkeiten nicht auch an eine neuen Generation von Forschenden vermittelt wird, kann sich dies in den deutschsprachigen Internationalen Beziehungen rächen.

Es stellt sich natürlich die Frage, wie viel Austausch effektiv über die Sprachgrenze geführt würde, wenn Blogs im deutschsprachigen Raum weiter verbreitet wären. Die wenigen existierenden Blogs nehmen durchaus Brückenfunktionen wahr. So können deutsche Debatten oder Forschungsresultate einem englischsprachigen Publikum vermittelt werden. Nicht zuletzt dank Debatten im Internet sind netzaffine Forschende oft viel besser über die Debatten und Diskussionen der Disziplin in den USA informiert, als über das, was in Deutschland geschieht. Ein digitaler Austausch via Internet findet, mit Ausnahme von privatem E-Mail-Verkehr natürlich, innerhalb des deutschsprachigen Raumes kaum statt. Dank Blogs könnten zudem der Stand der Forschung und die wichtigen Themen der Disziplin auf Deutsch einem viel breiteren Laienpublikum vermittelt werden. $\mathrm{Zu}$ guter Letzt kann alleine die Tatsache, dass in den Internationalen Beziehungen kaum auf Deutsch gebloggt wird, den Einstieg in die Welt der Blogs für den Nachwuchs in der Disziplin erschweren. Dies könnte in ein paar Jahren ein Wettbewerbsnachteil auf dem englischsprachigen akademischen Arbeitsmarkt sein, und ist es vielleicht mancherorts in Ausnahmefällen sogar schon.

Dies beeinflusst die Qualität des Diskurses im Fach wohl nur sehr bedingt negativ. Dies mag zumindest in einer kurzfristigen Perspektive zutreffend sein. Was jedoch der langfristige Effekt einer schlechteren Vernetzung sein könnte, ist weniger klar. Ein Abseitsstehen in einem System der beschleunigten Debatten mit schneller greifenden Korrekturmechanismen kann zum gravierenden Nachteil im globalen Ideenwettbewerb werden. Erste Schritte die Blogosphäre in existierende Institutionen einzubinden, finden schon statt. So fanden auf der Jahrestagung der International Studies Association (ISA) schon gut besuchte und prominent besetzte BloggingDiskussionsrunden statt (Arbia 2010). Auf der ISA 2013 wurde ein Blogging-Empfang organisiert und auch Preise für Bloggerinnen und Blogger vergeben (Nexon 2012b). Die Werbung für den Anlass und der Nominierungsprozess fanden vor allem in Blogs statt. Es waren wohl nicht viele Vertreterinnen oder Vertreter der deutschsprachigen IB anwesend. Es ist plausibel anzunehmen, dass die Bedeutung von Blognetzwerken bei solchen Anlässen zunehmen wird und es ist ein Fakt, dass viele deutschsprachige Vertreterinnen und Vertreter der IB nicht Teil davon sind.

Nun zum dritten Punkt: Die Kommunikation nach außen. In Zeiten von Haushaltsdisziplin und Sparmaßnahmen kann es sich ein Berufszweig, der sich stark über öffentliche Gelder finanziert, schlicht nicht leisten, die Öffentlichkeitsarbeit aus den Augen zu verlieren (die Coburn-Eingabe im US-Kongress habe ich schon erwähnt). Gerade im deutschsprachigen Raum fehlt es an einem öffentlichen Profil 
des Faches der Internationalen Beziehungen. Oft werden Vertreterinnen und Vertreter unsere Disziplin bestenfalls als Teil der TV-Expertokratie wahrgenommen, die bei weltpolitischem Bedarf vor den Kameras paradieren darf, unter der Bedingung, dass sie ihre Analyse in kleine verdaubare Häppchen verpackt. Hier bieten IB-Blogs eine Alternative und zwar mit einer besseren Ausgangslage als viele andere Sozialwissenschaften. Diese Chance gilt es unbedingt zu nutzen und unsere Kolleginnen und Kollegen auf der anderen Seite des Atlantiks scheinen dies schneller und besser verstanden zu haben.

Internationale Politik kann auf großes Interesse zählen. Es sind oft Themen, die emotionalisieren, die interessieren und zu denen viele politisch Gelegenheitsinteressierte eine Meinung haben. Mit Debatten vom Euro bis zu internationalem Terrorismus, vom Konflikt im Nahen Osten bis zur Kaschmir-Frage, existieren Themen zuhauf, die ein breites Publikum interessieren. Ein Indikator hierfür ist, dass in der Schweiz Referenden zu außenpolitischen Themen tendenziell oft höhere Partizipationsraten aufweisen als andere Themen und zwar seit der Staatsgründung 1848 (Goetschel et al. 2002: 100).

Die vierte Chance des Bloggens für die Internationale Beziehungen wird ebenfalls oft unterschätzt: Es ist eine Methode, Expertise und Evidenz aus der Forschung in den eigentlichen politischen Prozess einzuspeisen. Obwohl durchaus anzunehmen ist, dass zuständige Personen in den Außenministerien Blogs lesen, gibt es hierfür fast nur anekdotische Belege. Das Argument ist aber nicht neu. Schon 2004 stellten Drezner und Henry Farell die These auf, dass Blogs auch die außenpolitische Agenda bestimmen können und Politikentscheidungen beeinflussen (Drezner/Farell 2004). Es gibt durchaus Beispiele in der Geschichte des 20. Jahrhunderts, wo Meinungsbeiträge Politik direkt beeinflusst haben. ${ }^{8}$ Blogs sind die neuen Leitartikel und haben dasselbe Potential wie diese. Dass die Grenzen zwischen klassischen Kommentarspalten und Blogs immer mehr verwischen, sieht man an unzähligen Beispielen. Dass der Monkey Cage Blog nun bei der Washington Post zu Hause ist, oder ein Paul Krugman für die New York Times bloggt, sind zwei Beispiele, die immer häufigere Bezugnahme traditioneller Medien auf Blogs ist ein drittes.

Drezner und Farell nennen Beispiele, in denen Blogs für Medien mit einer viel größeren Reichweite als Themengeber funktionierten: Selbst wenn die Ideen von Blogs ausschließliche via traditionellen Journalismus in die Politik einfließen, bietet sich so trotzdem ein Weg, den politischen Diskurs mit zu prägen. Ein Beispiel, welches auch von Drezner und Farell aufgegriffen wird (Drezner/Farell 2004: 34), ist das Nahost-Blog eines Universitätsprofessors der Universität von Michigan. Dieser hatte Schwierigkeiten, Kommentare in klassischen Medien zu platzieren. Deshalb fing er 2002 an zu bloggen. Er erreichte 2013 nach eigenen Angaben vier Millionen Besucher (Cole 2014) und ist wegen des Blogs ein häufiger Gast in di-

8 Ein Beispiel hierfür wäre der berühmte Artikel von George Kennan The Sources of Soviet Conduct, in Foreign Affairs im Juli 1947, der die containment-Strategie gegenüber der Sowjetunion mitprägte. 
versen Radio- und TV-Sendungen der großen Netzwerke. Da ein Blog-Eintrag von ihm als eines der wenigen Ergebnisse bei einer Internet-Suche zu Muktada al-Sadr, dem irakischen Geistlichen, auftauchte, wurde er als Experte in den außenpolitischen Ausschuss des US-Senats eingeladen. Anekdotisch weiß man, dass er auch in den Außenministerien außerhalb der USA gelesen wird. Man kann sicherlich ohne Übertreibung sagen, dass Cole den politischen Diskurs nun durch seine Blogtätigkeit mit beeinflusst (Drezner/Farrell 2004: 34).

Die Blog-Angst, welche die Disziplin vor allem im deutschsprachigen Raum zu plagen scheint, führt so erneut zu einer verpassten Chance. Nicht nur könnten auf diese Weise Forschungsresultate in die Praxis einfließen, sondern es würde auch eine Möglichkeit bestehen, Politik über nationale Landesgrenzen hinaus zu formen. Ein vereintes Europa zum Beispiel bietet hierfür gute Möglichkeiten und einen öffentlichen politischen Raum, der traditionelle nationalstaatliche Grenzen überwindet. Die Schaffung eines europäischen Demos beginnt so vielleicht im Internet. Am Ende geht es aber um nicht weniger als die Relevanz der Disziplin. Diese riskiert, im deutschsprachigen Raum an Boden zu verlieren, wenn wir nicht bereit sind, Neuerungen wie Blogging in unsere Arbeit zu integrieren.

Wenn es so viele gute Argumente für das Bloggen gibt, könnte man sich fragen, woher denn diese Scheu in den Internationalen Beziehungen kommt, Bloggen als ein Instrument der Lehre und der Kommunikation zu akzeptieren. Ist es, wie die kürzlich in einem deutschsprachigen Soziologieblog aufgeworfene Frage suggeriert, vielleicht doch nur eine »anspruchsvolle Art der Zeitvergeudung« (Reichertz 2013)? Die klassischen drei Kritiken sind: Erstens, Blogs sind meist von schlechter Qualität und behandeln Triviales. Zweitens, Blogs sind eine Spielerei ohne Relevanz für die Disziplin und die Forschung im Allgemeinen. Drittens, einen Blog zu betreiben, ist Zeitverschwendung und die Zeit kann besser für klassische Forschungsarbeit genutzt werden (Reichertz 2013; Carpenter/Drezner 2010: 259). Hier soll kurz auf jede dieser drei Argumente eingegangen werden.

Ein häufig gehörter erster Einwand in akademischen Kreisen und den Medien ist, dass die Qualität von blog posts mangelhaft ist und Blogs als Medium sich vor allem mit Trivialitäten beschäftigen und oberflächlich seien. Doch wird da mit verschiedenen Maßstäben gemessen. Zum ersten Argument kann man sagen, dass niemand bestreitet, dass selbst der peer review-Prozess nicht verhindern kann, dass immer wieder auch qualitativ fragwürdige Artikel veröffentlicht werden. Außerdem sollen Blogs diesen Prozess nicht ersetzen, sondern bestenfalls ergänzen. Sie entsprechen dem Vortrag eines Gastes auf der Durchreise im Seminarraum. Eine schnelle und persönliche Art, Anregungen und Ideen für erste Entwürfe zu erhalten oder erste Versionen von Forschungsergebnissen vor einem Fachpublikum zu testen. Im Gegensatz zum Seminarraum-Publikum ist dieses im Idealfall breiter und spezialisierter, wenn gebloggt wird.

Der in der Qualitätskritik manchmal angefügte Trivialitätvorwurfs betreffend des Mediums selbst ist ebenfalls unangebracht. Niemand käme doch auf die Idee zu sagen, dass zum Beispiel The Economist eine unseriöse Quelle sein müsse, weil das Medium der Wochenmagazine mit überwältigender Mehrheit von Oberflächlich- 
keiten wie Schminktipps, Promi-Homestories und Sport geprägt ist. Es ist das Vertrauen in die Autorin respektive den Autor, welches am Ende die Glaubwürdigkeit determiniert. Hinter einem Blog steht normalerweise ein Name oder Pseudonym. Stimmt die Qualität, findet sich ein entsprechendes Publikum.

Wie steht es also um den zweiten Vorwurf, die angeblich mangelnde Relevanz von Blogs? Es entbehrt nicht einer gewissen Ironie, dass vor allem in den USA eine fast identische Debatte stattfindet, die sich darum dreht, inwiefern die Disziplin der Internationalen Beziehungen an und für sich ihre Relevanz verloren hat (Nye 2009; Walt 2009; 2005). Ein Vorschlag von Stephen Walt, wie man diesem »Kult der Irrelevanz« entgegenwirken könnte, ist, dass mehr Forschende bloggen sollten (Arbia 2009). Blogs sind also aus dieser Sicht nicht irrelevant, sondern ein potentielles Gegenmittel!

Dem Vorwurf der Irrelevanz haftet etwas Zirkuläres an und es macht ihn zur selbsterfüllenden Prophezeiung: Blogs sind nicht relevant für den Diskurs, weil sie als nicht relevant für den Diskurs betrachtet werden. Das Medium Blog führt keineswegs zwangsläufig in die Irrelevanz. Die Verweigerung, Blogs das Potential für Relevanz zuzugestehen, schadet am Ende so der ganzen Disziplin. Eine Divergenz zwischen verschiedenen Sprachgruppen, was als relevantes Medium zur Verbreitung von Ideen zu gelten hat, verhindert einen effizienten Informationsfluss innerhalb dieser. In anderen Disziplinen hat man die Zeichen der Zeit erkannt. Große seriöse wissenschaftliche Publikationen wie Nature und Science offerieren auf ihren Portalen Blogs und andere Web 2.0-Angebote, wie zum Beispiel Podcasts. In einem Editorial 2009 der Zeitschrift Nature mit dem Titel It's good to blog! wurden Wissenschaftlerinnen und Wissenschaftler in der gedruckten Ausgabe zum Bloggen aufgefordert (Nature 2009). Es gibt keinen Grund, warum ausgerechnet in den Internationalen Beziehungen gelten sollte: It's bad to blog.

Verschiedene Initiativen von englischsprachigen Blogs in den Internationalen Beziehungen und Politikwissenschaften zeigen, dass eine bessere Vernetzung mit traditionelleren akademischen Publikationsformen angestrebt wird. So plant zum Beispiel Daniel Nexon, der auf Duck of Minerva bloggt, seine Rolle als Redakteur des International Studies Quarterly mit seiner Bloggertätigkeit zu verbinden und Synergien zu erzeugen (Nexon 2013a; 2012a). Dasselbe Blog hat mit dem European Journal of International Relations auch ein Symposium organisiert, welches gleichzeitig im Blog und in der Printausgabe der Zeitschrift abgehalten wurde (Nexon 2013b; Wight et al. 2013). Das schon erwähnte politikwissenschaftliche Blog The Monkey Cage hat sich mit Verlagen geeinigt, dass publizierte Artikel für eine beschränkte Zeit frei zugänglich sein werden, nachdem sie im Blog vorgestellt wurden. So soll neue Forschung eine größere Öffentlichkeit finden und Diskussionen sollen geöffnet werden (Tucker 2013). Davon profitiert der Verlag natürlich ebenso wie die Autorinnen und Autoren.

Der dritte Vorwurf ist, dass Blogs Zeitverschwendung seien. Gerade junge Forscherinnen und Forscher sollen sich besser auf ihre Forschung konzentrieren, statt sich mit frivolen Blogeinträgen zu beschäftigen (Reichertz 2013, Carpenter/Drezner 2010: 259). Doch diese Sicht entspricht einem einseitigen Fokus und einem 
Missverstehen des Mediums. Die vielfältigen Vorteile zum Bewerben und Bekanntmachen der eigenen Forschungsarbeit wurden nun schon wiederholt erwähnt. Aber auch im Forschungsprozess selbst kann Bloggen nützlich sein. Es können Ideen zu spezifischen Problemen gesammelt oder schnell Referenzen gefunden werden (Carpenter und Drezner 2010: 261). Ein Blog zu betreiben, ist zudem eine gute Schreibübung. Klare Fachtexte zu verfassen, ist eine Qualität, die gerade in der akademischen Ausbildung leider zu oft vernachlässigt wird. Ideen auf ein paar Abschnitte zu reduzieren und für ein nicht spezialisiertes Publikum ohne Jargon zu schreiben, hilft Gedanken zu ordnen, kausale Argumente und postulierte Mechanismen auch im eigenen Kopf zu klären und explizit zu machen. Disziplin-relevante Debatten können viel zeitnaher und mit breiterer Partizipation geführt werden und sind so auch nützlicher. Von den vielen Angeboten, mit denen uns das Internet von der Arbeit abzuhalten versucht, ist ein Blog zu betreiben, die vermutlich denkbar produktivste Ablenkung.

\section{Die Neue Republik der Gelehrten: Ohne uns?}

Für diesen Beitrag wurde ich gebeten, über die Frage zu reflektieren, wie sich Blogs auf die Qualität des wissenschaftlichen Diskurses in den Internationalen Beziehungen auswirken. Basierend auf der dargestellten Situation sollte die Frage vielleicht umformuliert werden: Welchen Einfluss hat das Fehlen von deutschsprachigen Blogs auf die Qualität des wissenschaftlichen Diskurses in den Internationalen Beziehungen? Eingangs habe ich drei Thesen aufgestellt: Erstens, Blogs werden in Zukunft eine wichtige Rolle im wissenschaftlichen Diskurs spielen. Zweitens, die Disziplin der Internationalen Beziehungen eignet sich gut, wenn nicht sogar besser als andere sozialwissenschaftlichen Fächer, um vom Potential von Blogs zu profitieren. Drittens, die deutschsprachige IB-Welt ist im Begriff, eine wichtige Entwicklung zu verschlafen. Es stellt sich deshalb die Frage: Was tun?

In den Naturwissenschaften wird fleißig gebloggt. Es wächst eine neue Generationen heran, die Wissenschaftskommunikation betreibt und sich gleichzeitig besser und effektiver vernetzt, als das früher möglich war. Renommierte wissenschaftliche Zeitschriften wie Nature fordern Forschende zum Bloggen auf. Auch in den Internationalen Beziehungen zeichnet sich im englischsprachigen Raum ab, dass Blogs vermehrt in den Forschungsalltag integriert werden. Immer mehr informiert sich ein interessiertes Laienpublikum direkt über Wissenschaftlerinnen und Wissenschaftler, die Wissen verständlich und journalistisch aufarbeiten, statt sich durch Journalistinnen und Journalisten mit einem Faible für Wissenschaft vertreten zu lassen. Wir, die in der Disziplin der Internationalen Beziehungen forschen, werden nicht nur von der englischsprachigen Welt abgehängt, sondern auch von den Naturwissenschaften. Dies obwohl internationale Politik auf großes Interesse zählen kann und heute in den Medien durch (oft ad hoc) Expertinnen und Experten dominiert wird, eine Gruppe, die nicht selten methodisch unscharf und mit wenig Hintergrundwissen kommentiert. 
Solange Bloggen nicht als möglicher wertvoller Beitrag zur akademischen Arbeit gesehen wird, solange man bloggende Forscherinnen und Forscher der Zeitverschwendung verdächtigt, solange man Blogs in der Disziplin auf der Basis des genutzten Mediums alleine als irrelevant vom Tisch wischt, solange werden sich Teile des Fachs immer weiter ins Abseits manövrieren. Die Kluft zwischen den Naturwissenschaften und den Sozialwissenschaften einerseits und zwischen den deutschsprachigen und den englischsprachigen Welt anderseits wird so stetig anwachsen, wenn wir in dieser konservativen Grundhaltung verharren.

Die meisten existierenden IB-Blogs sind individuelle Initiativen. Es ist wünschenswert und notwendig, dass auf institutioneller Ebene ein Umdenken stattfindet. Bloggen sollte als eine akademische Tätigkeit anerkannt und in Bewerbungsverfahren gewürdigt werden. Lehrende sollten Blogs aktiv nutzen und nicht als etwas sehen, das wenn überhaupt nur als Hobby außerhalb des Vorlesungssaals eine Existenzberechtigung hat. Institutionen sollten Bloggen fördern und entsprechende Freiräume schaffen. Es darf nicht weiterhin auf eine exotische Freizeitbeschäftigung von technikverliebten Forscherinnen und Forschern reduziert werden, sondern sollte zu einer ausdrücklich erwünschten Tätigkeit und Teil des akademischen Pflichtenheftes werden. Nur wenn Blogs Teil der akademischen Arbeit sind, können wir den blog gap zum englischen Sprachraum verkleinern. Die Republik der Gelehrten 2.0 wird sonst ohne uns stattfinden. Das kann nicht im Interesse der Disziplin sein.

\section{Literatur}

Arbia, Ali 2009: IR: Wieviel Wissenschaft verträgt die Politik oder Warum Stephen Walt bloggt, in: http://scienceblogs.de/zoonpolitikon/2009/06/15/ir-wieviel-wissenschaft-vertragt-die-politik-oder-warum-stephen-walt-bloggt/; 10.03.2014.

Arbia, Ali 2010: Wissenschaftsblogging und Internationale Beziehungen - Eine Diskussionrunde, in: http://scienceblogs.de/zoonpolitikon/2010/02/25/wissenschaftsblogging-undinternationale-beziehungen-eine-diskussionrunde/; 10.03.2014.

Basken, Paul 2014: Senate Moves to Limit NSF Spending on Political Science, in: http://chronicle.com/article/Senate-Moves-to-Limit-NSF/138027/; 10.03.2014.

Bretterblog 2014: Webseite, in: http://bretterblog.wordpress.com; 07.04.2014.

Brownstein, Erica/Klein, Robert 2006: Blogs, in: Journal of College Science Teaching, 35: 6, 18-22.

Carpenter, Charli/Drezner, Daniel 2010: International Relations 2.0: The Implications of New Media for an Old Profession, in: International Studies Perspectives, 11: 3, 255272.

Chang, Daniel/Ge, Yuankai/Song, Shiwei/Coleman, Nicole/Christensen, Jon/Heer, Jeffrey 2009: Visualizing the Republic of Letters, in: http://www.stanford.edu/group/toolingup/ rplviz/papers/Vis_RofL_2009; 10.03.2014.

Churchill, Daniel, 2009: Educational Applications of Web 2.0: Using Blogs to Support Teaching and Learning, in: British Journal of Educational Technology, 40: 1, 179-183.

Cole, Juan 2014: Last Chance: Annual Informed Comment Fundraiser, in: http://www.juancole.com/2014/01/2013-informed-comment-fundraiser.html; 10.03.2014.

Drezner, Daniel/Farell, Henry 2004: Web of Influence, in: Foreign Policy, 145, 32-40.

Drezner Foreign Policy 2014: Webseite, in: http://drezner.foreignpolicy.com; 07.04.2014. 
Duck of Minerva 2014: Webseite, in: http://www.whiteoliphaunt.com; 07.04.2014.

Ferdig, Richard E./Trammell, Kaye D. 2004: Content Delivery in the >Blogosphere`, in: http:// thejournal.com/articles/2004/02/01/content-delivery-in-the-blogosphere.aspx; 10.03.2014.

Foreign Policy Magazine 2014: Webseite, in: http://www.foreignpolicy.com; 07.04.2014.

Goetschel, Laurent/Bernath, Magdalene/Schwarz, Daniel 2002: Schweizerische Aussenpolitik. Grundlagen und Möglichkeiten, Zürich.

Greenslade, Roy 2014: Academics Protest at Proposal to Ban them from Blogging, in: http:// www.theguardian.com/media/greenslade/2014/jan/30/blogging-higher-education; 10.03.2014.

Harrison, Chris 2007: Visualizations, Internet Maps, in: http://www.chrisharrison.net/ index.php/Visualizations/InternetMap; 10.03.2014.

Higdon, Judge/Topaz, Chad 2009: Blogs and Wikis as Instructional Tools: A Social Software Adaptation of Just-In-Time Teaching, in: College Teaching, 57: 2, 105-109.

Hypotheses 2014: Webseite, in: http://hypotheses.org; 07.04.2014.

Informed Comment 2014: Webseite, in: http://www.juancole.com; 07.04.2014.

International Relations and Security Network 2014: Webseite, in: http://isnblog.ethz.ch; 07.04.2014.

IR Blog 2014: Webseite, in: http://www.irblog.eu; 07.04.2014.

Kennan, George 1947: The Sources of Soviet Conduct, in: http://www.foreignaffairs.com/articles/23331/x/the-sources-of-soviet-conduct; 10.03.2014.

Krause, Steven D. 2005: Blogs as a Tool for Teaching, in: http://chronicle.com/weekly/v 51/ i42/42b03301.htm, 10.03.2014.

London School of Economics: Impact of Social Science (Webseite) 2014, in: http:// blogs.lse.ac.uk/impactofsocialsciences; 07.04.2014.

Monkey Cage 2014: Webseite, in: http://www.washingtonpost.com/blogs/monkey-cage; 07.04.2014.

LSE Public Policy Group 2011: Maximizing the Impacts of Your Research: A Handbook for Social Scientists, in: http://blogs.lse.ac.uk/impactofsocialsciences/the-handbook/; 10.03 .2014 .

Nature Editorial 2009: It's Good to Blog, in: http://www.nature.com/nature/journal/v 457/ n7233/full/4571058a.html; 10.03.2014.

Nexon, Daniel 2012a: Academic IR and the Information Age: Journals, in: http:// www.whiteoliphaunt.com/duckofminerva/2012/06/academic-ir-and-informationage.html; 10.03.2014.

Nexon, Daniel 2012b: Announcing the 2013 Blogging Reception and Awards at ISA, in: http://www.whiteoliphaunt.com/duckofminerva/2012/10/announcing-the-2013-blogging-reception-and-awards-at-isa.html; 10.03.2014.

Nexon, Daniel 2013a: Talking Academic Journals: Collecting Data, in: http://www.whiteoliphaunt.com/duckofminerva/2013/04/talking-academic-journals-collecting-data.html; 10.03.2014.

Nexon, Daniel 2013b: Special Event: The End of IR Theory, Symposium, in: http:// www.whiteoliphaunt.com/duckofminerva/2013/09/special-event-the-end-of-ir-theorysymposium.html; 10.03.2014.

Nye, Joseph 2009: Scholars on the Sidelines, in: http:/www.washingtonpost.com/wp-dyn/ content/article/2009/04/12/AR2009041202260.html; 10.03.2014.

PoliSciZurich 2014: Webseite, in: http://poliscizurich.wordpress.com; 07.04.2014.

Reichertz, Jo 2013: Zeitvergeudung, in: http://soziologie.de/blog/?p=870; 10.03.2014.

Research Blogging 2014: Webseite, in: http://researchblogging.com; 07.04.2014.

Scienceblogs 2014: Webseite, in: http://www.scienceblogs.de; 07.04.2014.

Scilogs 2014: Webseite, in: http://www.scilogs.de; 07.04.2014. 
Shema, Hadas/Bar-Ilan, Judit/Thelwall, Mike 2012: Research Blogs and the Discussion of Scholarly Information, in: http://www.plosone.org/article/info\%3Adoi\%2F10.1371\%2Fjournal.pone.0035869, 10.02.2014.

Sicherheitsbpolitik Blog 2014: Webseite, in: http://www.sicherheitspolitik-blog.de; 07.04.2014.

SozBlog 2014: Webseite, in: http://soziologie.de/blog/; 07.04.2014.

Straumsheim, Carl 2014: Is Blogging Unscholarly?, in: http://www.insidehighered.com/news/ 2014/01/29/international-studies-association-proposes-bar-editors-blogging; 10.03.2014.

The Conscience of a Liberal 2014: Webseite, in: http://krugman.blogs.nytimes.com; 07.04.2014.

Tucker, Joshua 2013: Collaboration with Journals and Presses to Highlight Recently Published Research Along with Ungated Links to Articles, in: http://themonkeycage.org/ 2013/05/24/collaboration-with-journals-and-presses-to-highlight-recently-published-research-along-with-ungated-links-to-articles/; 10.03.2014.

Walt, Stephen 2014: Foreign Policy (Webseite), in: http://walt.foreignpolicy.com; 07.04.2014.

Walt, Stephen 2009: The Cult of Irrelevance, in: http://walt.foreignpolicy.com/posts/ 2009/04/15/the_cult_of_irrelevance?wp_login_redirect $=0 ; 10.03 .2014$.

Walt, Stephen 2005: The Relationship Between Theory and Policy in International Relations, in: Annual Review of Political Science 8, 23-48.

Wight, Colin/Hansen, Lene/Dunne, Tim (Hrsg.) 2013: The End of IR Theory, Symposium in: European Journal of International Relations 19: 3, 405-425.

Wikipedia Deutschland 2014: Blog, in: https://de.wikipedia.org/wiki/Blog; 10.03.2014.

Wolfe-Simon, Felisa, et al. 2011: A Bacterium That Can Grow by Using Arsenic Instead of Phosphorus, in: Science 332: 6034, 1163-1166.

Zoon politicon 2014: Webseite, in: http://www.zoonpoliticon.ch; 07.04.2014.

Zoon politikon 2014: Webseite, in: http://www.scienceblogs.de/zoonpolitikon; 07.04.2014. 